International Mathematical Forum, 1, 2006, no. 4, 147 - 155

\title{
Certain Shifts on Banach Spaces of Formal Power Series ${ }^{1}$
}

\author{
K. Hedayatian \\ Department of Mathematics, Shiraz University \\ and \\ Institute for Studies in Theoretical Physics and Mathematics (IPM), Iran \\ hedayat@susc.ac.ir
}

\begin{abstract}
In this paper, we represent certain shifts on a Banach space as $M_{z}$, the operator of multiplication by the independent variable $z$, on some space of sequences. Also we will give necessary and sufficient conditions for the unitarily equivalence, similarity and quasisimilarity of such operators.
\end{abstract}

Mathematics Subject Classification: 47B37

Keywords: Banach space, shift, representation, power series, $H^{p}(\beta)$, unitarily equivalent, similar, quasisimilar.

\section{Introduction}

A bounded linear operator $\mathrm{A}$ on a Banach space $\mathcal{Y}$ is called a shift if

(i) A is an injective,

(ii) The range of $\mathrm{A}$ has codimension 1;

(iii) $\cap_{i=1}^{\infty} A^{i} y=\{0\}$.

$\mathrm{R}$. Crownover was the first to give a basis free definition of a shift on a Banach space. He has obtained some results concerning spectrum, and the commutant of such shifts [2]. In this connection, one can see [1], [3] and [5].

\footnotetext{
${ }^{1}$ This research was in part supported by a grant from IPM(No.83470040).
} 
Let $p>0$ and $\{\beta(n)\}$ denote a sequence of positive numbers such that $\beta(0)=1$. We consider the space of all sequences $f=\{\hat{f}(n)\}$ such that

$$
\|f\|_{p}^{p}=\sum_{n=0}^{\infty}|\hat{f}(n)|^{p} \beta(n)^{p}<\infty .
$$

We shall use the formal notation $f(z)=\sum_{n=0}^{\infty} \hat{f}(n) z^{n}$ whether or not the series converges for any complex values of $z$. Throughout this article, we consider the space $H^{p}(\beta)$ to be defined by

$$
H^{p}(\beta)=\left\{f: f(z)=\sum_{n=0}^{\infty} \hat{f}(n) z^{n},\|f\|_{p}<\infty\right\} .
$$

One of our goal in this article, is to study the representation of certain shifts on a Banach space as the operator $M_{z}$ on $H^{p}(\beta)$.

In all that follows we consider $1 \leq p<\infty$. Define the $\sigma$-finite measure $\mu$ on the nonnegative integers, $N_{0}$, by $\mu(K)=\sum_{n \in K} \beta(n)^{p}, K \subseteq N_{0}$. Since $H^{p}(\beta) \cong l^{p}(\mu)$, we conclude that $H^{p}(\beta)$ is, indeed, a Banach space. For some more information on the space $H^{p}(\beta)$ see [4] and [7].

It is natural to ask when the operators $\left(M_{z}, H^{p}(\beta)\right)$ and $\left(M_{z}, H^{p}(\alpha)\right)$ are unitarily equivalent, similar or quasisimilar. We would like to give necessary and sufficient conditions for unitarily equivalence, similarity and quasisimilarity of the operators $\left(M_{z}, H^{p}(\beta)\right)$ and $\left(M_{z}, H^{p}(\alpha)\right), 1 \leq p<\infty$. Furthermore, we show that if $A_{1}$ and $A_{2}$ are two injective unilateral weighted shifts on separable Hilbert spaces $\mathcal{H}_{1}$ and $\mathcal{H}_{2}$, respectively, and if there exist dense range operators $S: \mathcal{H}_{1} \rightarrow \mathcal{H}_{2}$ and $T: \mathcal{H}_{2} \rightarrow \mathcal{H}_{1}$ such that $A_{2} S=S A_{1}$ and $T A_{2}=A_{1} T$, then $A_{1}$ is similar to $A_{2}$ and vice-versa.

\section{Main Results}

It is worthwise remarking that if $\mathrm{A}$ be a shift on a Banach space $\mathcal{Y}$ then $\mathrm{A}$ is a closed range operator. This is a consequence of an easy automatic continuity result: If a finite codimensional linear subspace $\mathcal{X}$ of a Banach space $\mathcal{Y}$ is the range of a continuous linear mapping $N$ on $\mathcal{X}$, then it must be closed $[9$, Lemma 3.3]. It is known [2] that there is an element $y_{0} \in \mathcal{Y}$ with $\left\|y_{0}\right\|=1$ so that $\mathcal{Y}=<y_{0}>\oplus A \mathcal{Y}$ where $\left\langle y_{0}>\right.$ is a one-dimensional subspace of $\mathcal{Y}$ containing $y_{0}$.

Suppose $y \in \mathcal{Y}$. Then there exist a scalar $\hat{y}(0)$ and a vector $y_{1} \in \mathcal{Y}$ such that $y=\hat{y}(0) y_{0}+A y_{1}$. Similarly, there exist a scalar $\hat{y}(1)$ and a vector $y_{2} \in \mathcal{Y}$ 
such that $y_{1}=\hat{y}(1) y_{0}+A y_{2}$. So $y=\hat{y}(0) y_{0}+\hat{y}(1) A y_{0}+A^{2} y_{2}$. By induction, we obtain unique sequences $\{\hat{y}(n)\}_{n=0}^{\infty}$ and $\left\{y_{n}\right\}_{n=0}^{\infty}$ of scalars and vectors, respectively, such that for every $n$,

$$
y=\hat{y}(0) y_{0}+\hat{y}(1) A y_{0}+\cdots+\hat{y}(n) A^{n} y_{0}+A^{n+1} y_{n+1} .
$$

Equation (1) is called the Taylor formula for $y$, and the coefficients $\hat{y}(n)$ are called the Taylor coefficients of $y$.

Theorem 2.1. Let A be a shift on the Banach space $\mathcal{Y}=<y_{0}>\oplus A \mathcal{Y}$. Suppose that there exists $1 \leq p<\infty$ such that for every $y \in \mathcal{Y}$

(a) $\left\|\sum_{i=0}^{n} \hat{y}(i) A^{i} y_{0}\right\|^{p}=\sum_{i=0}^{n}\left\|\hat{y}(i) A^{i} y_{0}\right\|^{p}$ for all nonnegative integers $n$, where $A^{0}$ is the identity operator, and

(b) $\sum_{n=0}^{\infty}\left\|\hat{y}(n) A^{n} y_{0}\right\|^{p}<\infty$

Then $\mathrm{A}$ is unitarily equivalent to $M_{z}$ on $H^{p}(\beta)$ for a suitable choice of $\beta$. Moreover, such $p$ is unique.

Proof. Let $\beta(n)=\left\|A^{n} y_{0}\right\|$ and the linear map $B$ from $\mathcal{Y}$ into $H^{p}(\beta)$ be defined by $B y=\sum_{n=0}^{\infty} \hat{y}(n) z^{n}$. We first show that $B$ is an isometry. Let $y \in \mathcal{Y}$. The condition (b) implies the convergence of the sequence $\left\{A^{n+1} y_{n+1}\right\}$, appearing in (1). Define the linear map $S: \mathcal{Y} \times<y_{0}>\rightarrow \mathcal{Y}$ by $S\left(y, \alpha y_{0}\right)=\alpha y_{0}+$ Ay. Obviously, $S$ is bijective and continuous. Now, by the inverse mapping theorem, we conclude that $S^{-1}$ is also continuous, and so

$$
\|A y\|=\|S(y, 0)\| \geq \frac{\|(y, 0)\|}{\left\|S^{-1}\right\|}=c\|y\|,
$$

where $c=\frac{1}{\left\|S^{-1}\right\|}$. Thus, A is bounded below. This fact implies that $\operatorname{ran} A^{n}, n \geq$ 1 , is closed. Suppose that the sequence $\left\{A^{n+1} y_{n+1}\right\}_{n}$ converges to $y^{\prime} \in \mathcal{Y}$. Then $y^{\prime}$ is in $\bigcap_{n=1}^{\infty} A^{n} \mathcal{Y}$, and consequently, condition (iii) says that $y^{\prime}$ must be the zero vector. Therefore,

$$
\begin{aligned}
\|y\|^{p} & =\lim _{n \rightarrow \infty}\left\|\sum_{i=0}^{n} \hat{y}(i) A^{i} y_{0}\right\|^{p} \\
& \stackrel{(a)}{=} \lim _{n \rightarrow \infty} \sum_{i=0}^{n}\left\|\hat{y}(i) A^{i} y_{0}\right\|^{p} \\
& =\|B y\|^{p}
\end{aligned}
$$

which means that $B$ is an isometry.

Now, suppose that $f \in H^{p}(\beta)$, and $\varepsilon>0$ is given. Then there exists $N>0$ such that $\sum_{i=N}^{\infty}|\hat{f}(i)|^{p}\left\|A^{i} y_{0}\right\|^{p}<\varepsilon^{p}$. It results from this fact and condition (a) 
that the sequence $\left\{y_{n}\right\}_{n=0}^{\infty}$ defined by $y_{n}=\sum_{i=0}^{n} \hat{f}(i) A^{i} y_{0}$ is Cauchy and so is convergent. Suppose its limit is $y$. It is easily checked that $B y=f$, and so $B$ is surjective.

In the next step, we will first show that $M_{z}$ is bounded, and then $B A=$ $M_{z} B$. Put $M=\sup _{n} \frac{\beta(n+1)}{\beta(n)}$. It is clear that $M \leq\|A\|<\infty$. Therefore,

$$
\begin{aligned}
\|z f\|_{p}^{p} & =\sum_{n=0}^{\infty}|(z f)(n)|^{p} \beta(n)^{p} \\
& =\sum_{n=1}^{\infty}|\hat{f}(n-1)|^{p} \beta(n)^{p} \\
& =\sum_{n=0}^{\infty}|\hat{f}(n)|^{p} \beta(n+1)^{p} \\
& \leq M^{p} \sum_{n=0}^{\infty}|\hat{f}(n)|^{p} \beta(n)^{p} \\
& =\left.M^{p}|| f\right|_{p} ^{p} .
\end{aligned}
$$

Thus $M_{z}$ is bounded. Finally, if $y \in \mathcal{Y}$ then

$$
\begin{aligned}
B A y & =B\left(A\left(\sum_{i=0}^{\infty} \hat{y}(i) A^{i} y_{0}\right)\right) \\
& =B\left(\sum_{i=0}^{\infty} \hat{y}(i) A^{i+1} y_{0}\right) \\
& =\sum_{i=1}^{\infty} \hat{y}(i) z^{i+1} \\
& =M_{z}\left(\sum_{i=0}^{\infty} \hat{y}(i) z^{i}\right) \\
& =\left(M_{z} B\right)(y)
\end{aligned}
$$

which is the desired conclusion.

To complete the proof, it remains to show that $p$ is unique. Suppose $1 \leq$ $q<\infty$ and (a) and (b) hold for $q$ besides $p$. Thus, the operator $M_{z}$ on $H^{p}(\beta)$ is unitarily equivalent to the operator $\tilde{M}_{z}$ on $H^{q}(\beta)$ defined by $\tilde{M}_{z}(f)=z f$; consequently, there exists a unitary operator $U: H^{p}(\beta) \rightarrow H^{q}(\beta)$ such that $U M_{z}=\tilde{M}_{z} U$. Suppose $g$ is in $H^{p}(\beta)$ so that $U g=1$. Then

$$
1=U g=U\left(\sum_{n=0}^{\infty} \hat{g}(n) z^{n}\right)=\sum_{n=0}^{\infty} \hat{g}(n) z^{n} U 1 .
$$


It is clear from the definition of the norm that the mapping $f \longmapsto \hat{f}(0)$ is bounded, thus $1=\hat{g}(0)(U 1)(0)$. Furthermore, $U$ is an isometry, and so $\sum_{n=0}^{\infty}|\hat{g}(n)|^{p} \beta(n)^{p}=1$. Since $\beta(0)=1$, we observe that $|\hat{g}(0)| \leq 1$. Hence

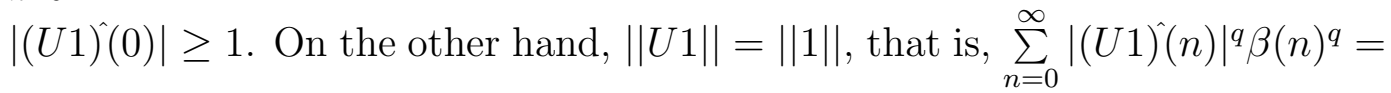
1 , and so $|(U 1) \hat{(0)}| \leq 1$; consequently, $|(U 1) \hat{(0)}|=1$. Moreover, since $1=$ $\|U 1\|^{q}=\sum_{n=0}^{\infty}\left|(U 1)^{\hat{(}(n)}\right|^{q} \beta(n)^{q}$, we have $(U 1)^{\hat{(}(n)}=0, n \geq 1$. Putting $U 1=c$, for some constant $c$, we see that

$$
2^{1 / p}=\left\|1+\frac{z}{\beta(1)}\right\|=\left\|U\left(1+\frac{z}{\beta(1)}\right)\right\|=\left\|c+\frac{z}{\beta(1)} c\right\|=2^{1 / q},
$$

which results that $p=q . \diamond$

Example 2.2. Let $p=2$ and suppose that $\mathrm{A}$ is a shift isometry on $H^{p}(\beta)$. Then $H^{p}(\beta)=\bigoplus_{i=0}^{\infty}<A^{i} y_{0}>$ and the uniquness of the Taylor coefficients implies that $\mathrm{A}$ is unitary equivalent to $M_{z}$ on $H^{p}(\beta)$.

Recall that if $A_{1}$ and $A_{2}$ are operators on Banach spaces $X_{1}$ and $X_{2}$, respectively, then $A_{1}$ and $A_{2}$ are unitarily equivalent, if there is an isomorphism (i.e., surjective linear isometry) $U: X_{1} \rightarrow X_{2}$ such that $U A_{1} U^{-1}=A_{2}$.

Theorem 2.3. Two operators $\left(M_{z}, H^{p}(\beta)\right)$ and $\left(M_{z}, H^{p}(\alpha)\right)$ are unitarily equivalent if and only if there exists a constant $c$ such that $\beta(n)=|c| \alpha(n)$, for every nonnegative integer $n$.

Proof. First, suppose that $U: H^{p}(\beta) \rightarrow H^{p}(\alpha)$ is an isomorphism such that $U M_{z}=M_{z} U$. Then for every $k>0$,

$\left\|U 1+z^{k}\right\|^{p}-\left\|U 1-z^{k}\right\|^{p}=\left\|U 1+z^{k} U g\right\|^{p}-\left\|U 1-z^{k} U g\right\|^{p}($ where $U g=1$ )

$$
\begin{aligned}
& =\left\|U\left(1+z^{k} g\right)\right\|^{p}-\left\|U\left(1-z^{k} g\right)\right\|^{p} \\
& =\left\|1+z^{k} g\right\|^{p}-\left\|1-z^{k} g\right\|^{p}=0 .
\end{aligned}
$$

On the other hand,

$$
\left\|U 1 \pm z^{k}\right\|^{p}=\sum_{n \neq k}\left|(U 1)^{\hat{(}(n)}\right|^{p} \beta(n)^{p}+\mid(U 1)^{\hat{(}(k) \pm\left. 1\right|^{p} \beta(k)^{p} .}
$$

So $\left|(U 1)^{\hat{(}}(k)+1\right|^{p} \beta(k)^{p}-\left|(U 1)^{\hat{(}}(k)-1\right|^{p} \beta(k)^{p}=0$. Since $\beta(k)>0$ it follows that

$$
|(U 1) \hat{(k)}+1|=|(U 1) \hat{(k)}-1|
$$


The same argument shows that

$$
|(U 1) \hat{(}(k)+i|=|(U 1) \hat{(}(k)-i| .
$$

Formula (3) combined with (4) shows that $(U 1)^{\wedge}(k)=0$, for every $k>0$. Therefore, $U 1=c$ for some constant $c$. This, in turn, implies that $U z^{n}=c z^{n}$ for all $n \geq 0$. Hence

$$
\beta(n)=\left\|z^{n}\right\|_{H^{p}(\beta)}=\left\|U z^{n}\right\|_{H^{p}(\alpha)}=|c| \alpha(n) \quad \forall n \geq 0 .
$$

For the converse, define $U: H^{p}(\beta) \rightarrow H^{p}(\alpha)$ by $U f=c f$. It can be easily verified that $U$ is an isomorphism and $U M_{z}=M_{z} U$.

Definition 2.4. If $A_{1}$ and $A_{2}$ are operators on Banach spaces $X_{1}$ and $X_{2}$, respectively, then $A_{1}$ is similar to $A_{2}$ if there is an invertible operator $S$ : $X_{1} \rightarrow X_{2}$ such that $S A_{1}=A_{2} S$. The operators $A_{1}$ and $A_{2}$ are quasisimilar if there are operators $S_{12}: X_{2} \rightarrow X_{1}$ and $S_{21}: X_{1} \rightarrow X_{2}$ satisfying the following conditions for $i, j=1,2$ :

(a) $\operatorname{ker} S_{i j}=\{0\}$ and $\operatorname{ran} S_{i j}$ is dense;

(b) $S_{i j} A_{j}=A_{i} S_{i j}$

(take $S_{11}$ and $S_{22}$ to be the identity operators). Operators satisfying (a), are said to be quasi-affinities.

Lemma 2.5. Let $S: H^{p}(\beta) \rightarrow H^{p}(\alpha)$ be an operator such that $M_{z} S=S M_{z}$. If $\operatorname{ran} S$ is dense then there exists a constant $c$ such that

$$
0<c \leq \frac{\beta(n)}{\alpha(n)} \quad \text { for all } n \geq 0 \text {. }
$$

Proof. First, note that if $(S 1) \hat{(}(0)=0$, then

$$
\left(S z^{n}\right)^{\hat{(}}(0)=\left(z^{n} S 1\right) \hat{(0)}=0 \text { for all } n \geq 0 .
$$

Since the mapping $f \longmapsto \hat{f}(0)$ is bounded we conclude that $(S f)^{\hat{n}}(0)=0$ for every $f$ in $H^{p}(\beta)$. Furthermore $\operatorname{ran} S$ is dense in $H^{p}(\alpha)$, and so there would exist a sequence $\left\{f_{n}\right\}$ in $H^{p}(\beta)$ such that $S f_{n}$ converges to 1 . So $\left(S f_{n}\right)^{\hat{\gamma}}(0) \rightarrow 1$, contradicting the fact that $\left(S f_{n}\right) \hat{(0)}=0$ for every nonnegative integer $n$. Thus, we see that $(S 1) \hat{(}(0) \neq 0$. Now

$$
\begin{aligned}
\alpha(n)|(S 1) \hat{(}(0)| & =\alpha(n) \mid\left(z^{n} S 1 \hat{)}(n) \mid\right. \\
& =\alpha(n)\left|\left(S z^{n}\right) \hat{(n)}\right| \\
& \leq\left\|S z^{n}\right\| \leq\|S\| .\left\|z^{n}\right\|=\|S\| \beta(n) \text { for all } n \geq 0
\end{aligned}
$$


Hence if we put $c=\frac{\left|(S 1)^{\hat{r}}(0)\right|}{\|S\|}$ then $0<c \leq \frac{\beta(n)}{\alpha(n)}$ for every $n \geq 0$. $\diamond$

We have already seen that when the operators $\left(M_{z}, H^{p}(\beta)\right)$ and $\left(M_{z}, H^{p}(\alpha)\right)$ are unitarily equivalent. Now, we pose the question of similarity of these operators.

Theorem 2.6. The operator $\left(M_{z}, H^{p}(\beta)\right)$ is similar to $\left(M_{z}, H^{p}(\alpha)\right)$ if and only if there exist positive constants $C_{1}$ and $C_{2}$ such that $0<C_{1} \leq \frac{\beta(n)}{\alpha(n)} \leq C_{2}$ for every nonnegative integer $n$.

Proof. Suppose $\left(M_{z}, H^{p}(\beta)\right)$ is similar to $\left(M_{z}, H^{p}(\alpha)\right)$. Then there is an invertible operator $S: H^{p}(\beta) \rightarrow H^{p}(\alpha)$ such that $S M_{z}=M_{z} S$; or equivalently $M_{z} S^{-1}=S^{-1} M_{z}$. By Lemma 3.3, there exist constants $C_{1}$ and $C_{2}$ such that

$$
0<C_{1} \leq \frac{\beta(n)}{\alpha(n)} \text { and } 0<\frac{1}{C_{2}} \leq \frac{\alpha(n)}{\beta(n)},
$$

for all $n \geq 0$. For the converse, suppose that there exist positive constants $C_{1}$ and $C_{2}$ so that $0<C_{1} \leq \frac{\beta(n)}{\alpha(n)} \leq C_{2}$ for every $n \geq 0$. It follows that $H^{p}(\alpha)$ and $H^{p}(\beta)$ coincide as sets with equivalent norms. Take for $S$ the identity operator. $\diamond$

Corollary 2.7. Suppose $S: H^{p}(\beta) \rightarrow H^{p}(\alpha)$ and $T: H^{p}(\alpha) \rightarrow H^{p}(\beta)$ be two dense range operators such that $M_{z} S=S M_{z}$ and $T M_{z}=M_{z} T$. Then $\left(M_{z}, H^{p}(\beta)\right)$ is similar to $\left(M_{z}, H^{p}(\alpha)\right)$.

Proof. By Lemma 2.5, there exist constants $c$ and $d$ such that $0<c<\frac{\beta(n)}{\alpha(n)}$ and $0<d<\frac{\alpha(n)}{\beta(n)}$ for every $n \geq 0$. Put $C_{1}=c$ and $C_{2}=\frac{1}{d}$, and apply Theorem 2.6. $\diamond$

Another immediate consequence is the following.

Corollary 2.8. The operators $\left(M_{z}, H^{p}(\beta)\right)$ and $\left(M_{z}, H^{p}(\alpha)\right)$ are similar if and only if they are quasisimilar.

We recall that a unilateral weighted shift operator A on a separable Hilbert space $\mathcal{H}$ is an operator that maps each vector in some orthonormal basis $\left\{e_{n}\right\}_{n=0}^{+\infty}$ into a scalar multiple of the next vector; i.e., $A e_{n}=w_{n} e_{n+1}$. The operator $\mathrm{A}$ is injective if and only if $w_{n} \neq 0$ for every $n$. It is known that every injective unilateral weighted shift can be represented as $M_{z}$ on $H^{2}(\beta)$ for a suitable choice of $\beta$; see [8] . In this view, Corollary 2.7 provides the following.

Corollary 2.9. Let $A_{1}$ and $A_{2}$ be two injective unilateral weighted shift on the Hilbert spaces $\mathcal{H}_{1}$ and $\mathcal{H}_{2}$. If there exist dense range operators $S: \mathcal{H}_{1} \rightarrow \mathcal{H}_{2}$ 
and $T: \mathcal{H}_{2} \rightarrow \mathcal{H}_{1}$ such that $A_{2} S=S A_{1}$ and $T A_{2}=A_{1} T$, then $A_{1}$ is similar to $A_{2}$.

It is known that if two injective unilateral weighted shifts are quasisimilar then they are similar. We can easily get this result as a conclusion of Corollary 2.9. Also it follows from [6, Theorem 4.6.] that if $A_{1}$ and $A_{2}$ are two injective unilateral weighted shifts on a Hilbert space $\mathcal{H}$ and if $S$ and $T$ are two bounded surjective operators on $\mathcal{H}$ such that $A_{2} S=S A_{1}$ and $T A_{2}=A_{1} T$ then $\sigma\left(A_{1}\right)=$ $\sigma\left(A_{2}\right)$. Since similarity preserves the spectrum and the various parts of the spectrum, we also get the following from Corollary 2.9.

Corollary 2.10. Let $A_{1}$ and $A_{2}$ be two injective unilateral weighted shifts on Hilbert spaces $\mathcal{H}_{1}$ and $\mathcal{H}_{2}$. Suppose there exist operators $S: \mathcal{H}_{1} \rightarrow \mathcal{H}_{2}$ and $T: \mathcal{H}_{2} \rightarrow \mathcal{H}_{1}$ which have dense range, and satisfy $A_{2} S=S A_{1}$ and $T A_{2}=A_{1} T$. Then $\sigma\left(A_{1}\right)=\sigma\left(A_{2}\right)$.

\section{REFERENCES}

1. Araujo, J. and Font, J. J.; Isometric shifts and metric spaces, Monatshefte für Mathematik volume 134 Issue 1 (2001) P. 1-8.

2. Crownover, R. M.; Commutants of shifts on Banach spaces, Michigan Math. J. 19(1972), 233-247.

3. Gutek, A., Hart, D., Jamison, J. and Rajagopalan, M.; Shift Operators on Banach spaces, Journal of Functional Analysis 101, 97-119 (1991).

4. Hedayatian, K.; On Cyclicity in the Space $H^{p}(\beta)$, Taiwanese Journal of Mathematics, Vol. 8, No.3, pp. 429-442, September 2004.

5. Holub, J.R.; On Shift Operators, Canad. Math. Bull. 31 (1988) 85-94.

6. Seddighi, K., Hedayatian, K.; Operators intertwining invertibly weighted shifts, Bull. Iranian Math. Soc., 19 (1993), 1-14.

7. Seddighi, K. Hedayatian, K. and Yousefi, B.; Operators acting on certain Banach spaces of analytic functions, Internat. J. Math. and Math. Sci. Vol. 18 No. 1 (1995) 107-110.

8. Shields, A.; Weighted shift operators and analytic function theory, American Mathematical Society,Providences, R. I.,1974,P.49-128. 
9. Sinclair, A. M.; Automatic Continuity of Linear Operators, London Math. Soc. Lecture Note Ser. 21, Cambridge Univ. Press, 1976.

Received: July 4, 2005 